\title{
Raman lasers for trace gas detection
}

\section{Zesch, Christoph, Schrader, Sigurd, Prosposito, Paolo, Lux, Oliver, Eichler, Hans}

Christoph Zesch, Sigurd Schrader, Paolo Prosposito, Oliver Lux, Hans J. Eichler, "Raman lasers for trace gas detection," Proc. SPIE 11042, XXII International Symposium on High Power Laser Systems and Applications, 110420M (3 January 2019); doi: 10.1117/12.2522463

SPIE Event: XXII International Symposium on High Power Laser Systems and Applications, 2018, Frascati, Italy 


\title{
Raman lasers for trace gas detection
}

\author{
Christoph Zesch ${ }^{1,2,4}$, Sigurd Schrader ${ }^{2}$, Paolo Prosposito ${ }^{1}$, Oliver Lux ${ }^{3}$, Hans J. Eichler ${ }^{4}$ \\ ${ }^{1}$ University of Rome "Tor Vergata", Via Cracovia n.50, 00133 Rome; \\ ${ }^{2}$ Technical University of Applied Sciences Wildau, Hochschulring 1, 15745 Wildau; \\ ${ }^{3}$ Institute for Atmospheric Physics, German Aerospace Center (DLR), \\ Münchener Straße 20, 82234 Weßling-Oberpfaffenhofen; \\ ${ }^{4}$ Institute for Optics and Atomic Physics, TU Berlin, Straße des 17. Juni 135, 10623 Berlin
}

\begin{abstract}
The emission of commercial solid-state lasers can be shifted by Raman lasers to selected wavelengths suitable for trace gas detection. High power Raman lasers based on $\mathrm{Ba}\left(\mathrm{NO}_{3}\right)_{2}$, diamond and silicon are useful to detect $\mathrm{CO}_{2}, \mathrm{O}_{3}$, and $\mathrm{H}_{2} \mathrm{O}$. Such lasers are based on the physical effect of stimulated Raman scattering. A new SRS spectrum of a recently investigated Raman crystal $\left(\mathrm{LuAlO}_{3}\right)$ is shown confirming previous results.
\end{abstract}

Keywords: Stimulated Raman Scattering, Raman lasers, Barium nitrate, diamond, silicon, trace gas detection

\section{INTRODUCTION}

Fifty years have passed since the discovery ${ }^{1}$ and subsequent correct identification ${ }^{2}$ of stimulated Raman scattering (SRS). In recent years, this process has established itself as a versatile method to generate new laser wavelengths resulting from a photon-phonon-interaction which leads to down- (Stokes) or up-shifting (anti-Stokes) of the pump laser frequency. The size of the frequency shift depends on the Raman-active material and the excitability of their SRS-promoting vibrationmodes. A large number of various Raman-active media has been investigated in the last decades shown in Figure 1 . The differences between these materials are on the one hand the variety of the wavelength shifts and on the other hand, the associated Raman gain coefficient, which is related to the conversion efficiency.

Prominent Raman crystals include $\mathrm{Ba}\left(\mathrm{NO}_{3}\right)_{2}$ and other nitrates, $\mathrm{KGW}$ and other tungstates, $\mathrm{YVO}_{4}$ and other vanadates as well as diamond. These crystals exhibit lattice vibrations with wave numbers from $80 \mathrm{~cm}^{-1}$ to $3150 \mathrm{~cm}^{-1}$, shifting the incident laser wavelength to higher or lower values. Recently, SRS was observed in the laser crystal $\mathrm{LuAlO}_{3}$ with three SRS-active phonon modes $^{3}$, see section 2.

Selective amplification of one particular spectral line generated through SRS is possible by placing the Raman crystal into a frequency-selective optical resonator, whose optical feedback is selective for only one Stokes- or anti-Stokes component. This configuration strongly decreases the SRS threshold and results in low-divergent laser radiation at the desired wavelength. Raman lasers can be used in many applications, e.g. differential absorption LIDAR systems (DIAL) to detect trace gases like carbon dioxide $\left(\mathrm{CO}_{2}\right)$, ozone $\left(\mathrm{O}_{3}\right)$ or water vapor $\left(\mathrm{H}_{2} \mathrm{O}\right)$.

Following this approach, a substantial extension of the spectral coverage of solid-state lasers has been achieved over the last five decades. Various pumping schemes and resonator designs have been investigated focusing on good conversion efficiency, high spatial beam quality and high pulse energy of the output beam. Vanadates, tungstates, diamond and barium nitrate crystals represent most common Raman materials for efficient laser operation. $\mathrm{Ba}\left(\mathrm{NO}_{3}\right)_{2}$ has a high gain coefficient $\left(g_{\mathrm{S}}=11 \mathrm{~cm} / \mathrm{GW}\right)$, good mechanical stability and is commercially available in large sample sizes. This crystal is often used for Raman lasers. A disadvantage is its high thermo-optic coefficient $(\mathrm{d} n / \mathrm{d} T)$ which leads to significant thermal lensing affecting the resonator stability. Accurate compensation of the induced thermal lens is necessary to ensure high conversion efficiency. According to the Raman shift of $\mathrm{Ba}\left(\mathrm{NO}_{3}\right)_{2}$, which is $1047.4 \mathrm{~cm}^{-1}$, selective amplification of the third Stokes order results in laser radiation around $1.599 \mu \mathrm{m}$ when pumping at $1.064 \mu \mathrm{m}$ wavelength with a Nd:YAG laser. ${ }^{4}$

XXII International Symposium on High Power Laser Systems and Applications, edited by Paolo Di Lazzaro Proc. of SPIE Vol. 11042, 110420M · @ 2019 SPIE · CCC code: 0277-786X/19/\$18 · doi: 10.1117/12.2522463 


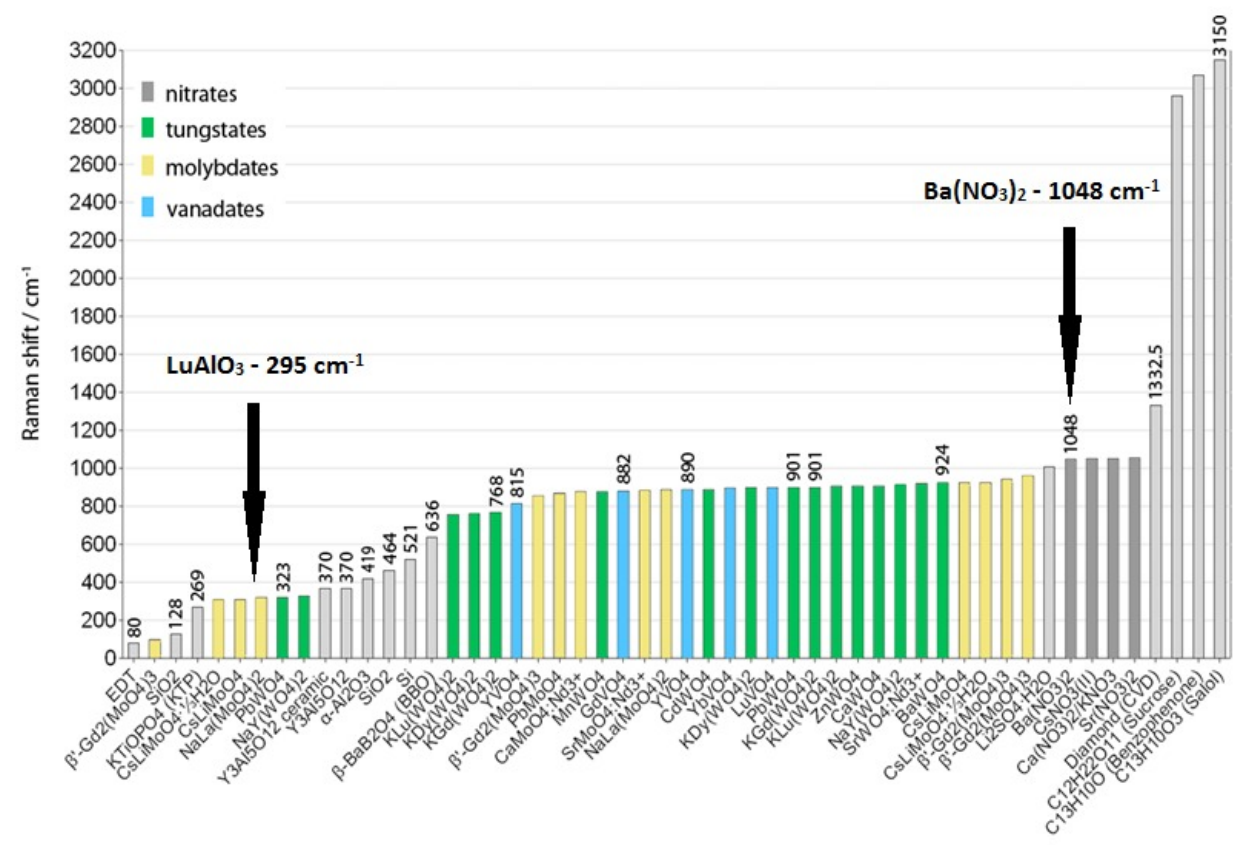

Figure 1. Raman shifts of the most known SRS-active crystalline media which were investigated in the last decades. ${ }^{5}$

Laser sources in this "eye-safe" spectral region have found increasing interest in LIDAR systems for trace gas detection. For example, space-borne DIAL systems for long-range measurements of $\mathrm{CO}_{2}$ concentration in the atmosphere require pulsed lasers with nanosecond pulse width in the wavelength between 1.570 and $1.613 \mu \mathrm{m} .{ }^{6}$ The DIAL technique involves two neighboring laser wavelengths, which are chosen such that one coincides with an absorption line of the measured gas molecule while the other one is only weakly absorbed. If these two wavelengths are transmitted into the atmosphere and the backscattering radiation is detected as a function of time, the differential absorption of the two lines provides information on the concentration of the investigated gas molecule species along the laser beam path.

Another trace gas molecule species whose concentration can be determined by the DIAL technique is ozone. Here, the barium nitrate crystal is pumped by $532 \mathrm{~nm}$ wavelength (frequency-doubled Nd:YAG laser) and the selective amplification of the second Stokes order leads to $598 \mathrm{~nm}$. Frequency doubling of this wave produces UV radiation at about $300 \mathrm{~nm}$ wavelength, which allows measuring the ozone altitude distribution both in the troposphere and stratosphere.

The DIAL technique requires laser sources with high average output power combined with an excellent beam quality $\left(M^{2}<2\right)$. Energy scaling requires larger pump beam diameters, resulting in a larger number of excited transversal modes, and hence a larger $M^{2}$ factor of the generated radiation. One possible solution can be found in an effect called Raman beam cleanup (RBC), which also takes place by using Raman amplifiers. In 1999, Murray et al. investigated the effect of RBC in Raman lasers both theoretically and practically. They showed that RBC is a direct consequence of four-wave-mixing and gain-guiding. ${ }^{7}$

Over the last ten years, diamond has been demonstrated as an excellent material for high-power Raman frequency conversion, particularly due to its beneficial thermo-mechanical properties. ${ }^{8}$ For instance, Lux at al. reported a narrowband and tunable diamond Raman laser generating "eye-safe" radiation suitable for water vapor detection. ${ }^{9}$ Efficient frequency conversion of a tunable pump laser operating from 1063 to $1066 \mathrm{~nm}$ to the second-order Stokes component was achieved in an external standing-wave cavity. The reached output power was high with $7 \mathrm{~W}$ in the wavelength range from 1483 to $1488 \mathrm{~nm}$.

A further Raman material is silicon producing wavelengths in the "eye-safe" region to detect trace gas concentrations. In 2008, Rong et al. reported a cascaded silicon Raman laser. ${ }^{10}$ Using a pump laser at $1.550 \mu \mathrm{m}$, selective amplification of the second Stokes order leads to $1.848 \mu \mathrm{m}$. This wavelength can be used in DIAL systems to measure water vapor concentrations. 


\section{STIMULATED RAMAN SPECTROSCOPY}

Stimulated Raman scattering is initiated by focusing the pump laser beam into the Raman-active crystal. The generated frequency-shifted light is collected by further lenses and detected with a spectrometer in combination with a CCD-camera. The Stokes intensity shows an exponential dependence on the length of the crystal and its Raman gain coefficient:

$$
I_{s}(x)=I_{s}(0) \exp \left(g_{s} I_{L} x\right)
$$

In the last year, we investigated the new Raman crystal lutetium aluminate $\left(\mathrm{LuAlO}_{3}\right)$. The cylindrical crystal was provided by Prof. A.A. Kaminskii and he published first results in 2016, presumably with another crystal. ${ }^{3}$ In figure 2, the SRS wavenumber spectrum is shown by pumping with the fundamental Nd:YAG laser beam of $1064 \mathrm{~nm}$. The higher frequency spectrum exhibits the anti-Stokes lines and the lower ones are the Stokes-lines. The mean spacing of the generated spectral lines is approximately $295 \mathrm{~cm}^{-1}$ with a standard deviation of about $3 \mathrm{~cm}^{-1}$. This phonon frequency corresponds with the measurement of Kaminskii and Lux with an average Raman shift of $297 \mathrm{~cm}^{-1}$. The other two SRS lines have not been detected by us because of the special crystal orientation.

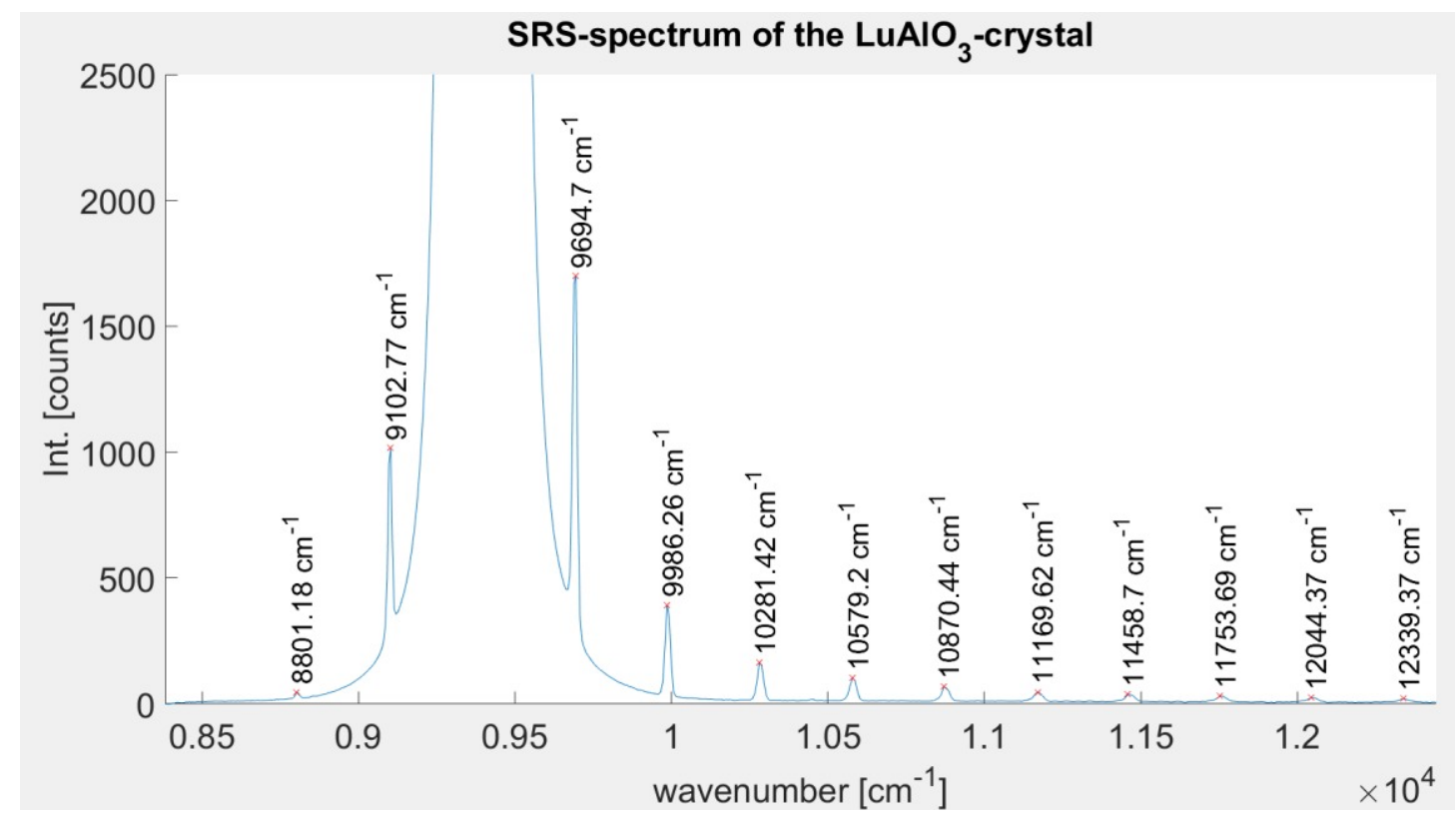

Figure 2. SRS-wavenumber spectrum of the $\mathrm{LuAlO}_{3}$-crystal excited at $1064 \mathrm{~nm}$ by a $\mathrm{Nd}:$ YAG laser.

\section{LIDAR SYSTEMS FOR TRACE GAS DETECTION}

As mentioned above, Raman lasers can be employed in LIDAR systems. To measure the concentration of trace gases in the atmosphere by means of the DIAL-technique, where two neighboring laser wavelengths are used. One is located in an absorption band of the investigated gas (on-line radiation), and the other one is only weekly absorbed (off-line radiation). If these two wavelengths are transmitted into the atmosphere the backscattering radiation is detected by a function of time. The differential absorption of this two lines provides information about the concentration of the investigated gas molecule. 


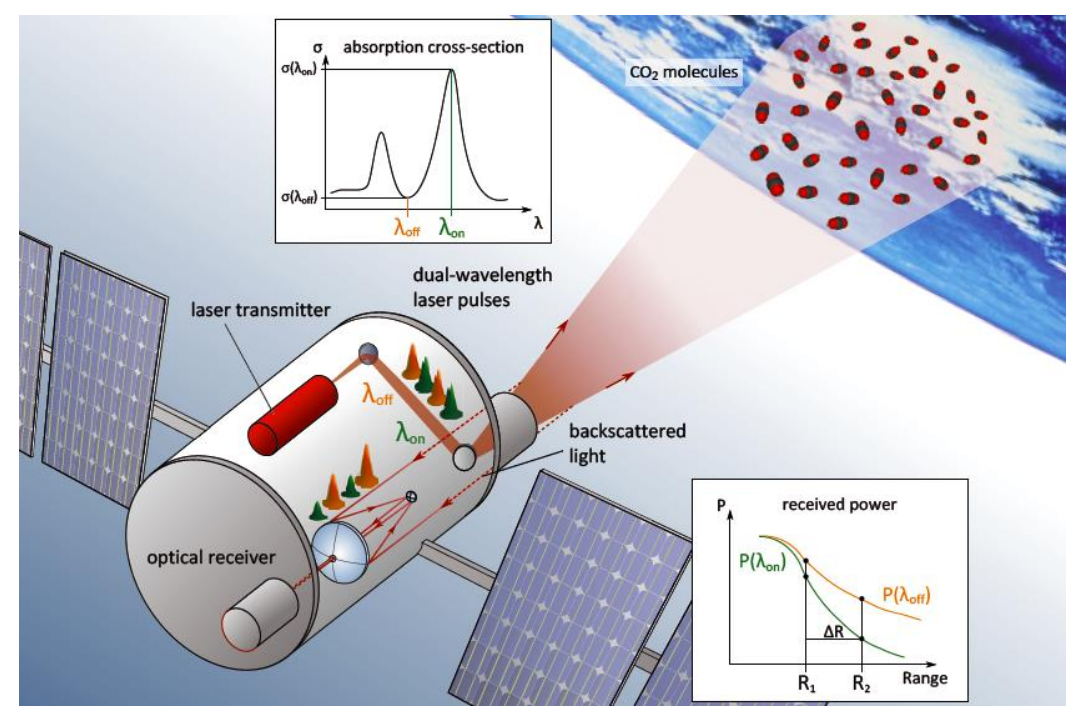

Figure 3. Principle setup of a space-born Differential Absorption LIDAR (DIAL) system. The insets show the absorption cross-section of the detected gas species and a typical measurement of the received power in dependence upon the scanned range for on- and off-wavelength. ${ }^{5}$

\section{SIMULTANEOUS RAMAN AMPLIFIER FOR OZONE DETECTION}

A well-known trace gas molecule species related to the greenhouse effect is ozone $\left(\mathrm{O}_{3}\right)$, so it is very important to control its atmospheric concentration. Therefore, a current research project in Wildau funded by the German Research Foundation aims to develop a simultaneous Raman amplifier system for ozone detection. In 2012, Dong et al. proved practically the effect of simultaneous Raman amplification in fibers. ${ }^{11}$ No investigations of simultaneous Raman amplifications of several Stokes waves in crystals have been performed so far. In the frame of this approach for $2^{\text {nd }}$ Stokes Raman amplification, both the $1^{\text {st }}$ and $2^{\text {nd }}$ Stokes seed beams with low energy and low value of $\mathrm{M}^{2}$ factor are input into a Raman amplifier. First, the energy of the pump radiation is converted to a low divergent $1^{\text {st }}$ Stokes wave. As the intensity of this wave become high enough the $2^{\text {nd }}$ Stokes amplification starts and the energy transfer from $1^{\text {st }}$ to the $2^{\text {nd }}$ Stokes wave occurs.

Two Stokes barium nitrate Raman lasers, pumped by a frequency-doubled Nd:YAG laser at $532 \mathrm{~nm}$, are used. The second Stokes wavelength amounts to $598 \mathrm{~nm}$ and the frequency doubling of this wave produces UV-radiation at a wavelength of approximately $300 \mathrm{~nm}$, which is absorbed by the ozone molecule. To use this radiation in LIDAR systems, high pulse energy with a good beam quality is needed, this means a low value of the propagation factor $M^{2}$ should be reached. However, these two requirements are difficult to be satisfied simultaneously. The incompatibility between good beam quality and high output pulse energy can be explained with the following. To generate Stokes radiation with a low value of $M^{2}$ it is necessary to use a pump beam with a small diameter. In this case, a small number of transversal modes can be generated, which leads to a good beam quality. On the other hand, a small beam diameter means high intensity of pump radiation and can cause disruption of the Raman crystal and the resonator mirrors. Hence, only pump beams with small energy should be used, which leads to small energy of the output Stokes pulse. The predicted solution can be found in the effect of Raman beam-cleanup ${ }^{6}$, which allows for a better beam quality. The theoretical cross-section of the depleted pump beam, and of the $1^{\text {st }}$ and $2^{\text {nd }}$ Stokes beams are calculated, and the result is shown in figure 4 . The intensities are normalized to the value of the pump intensity. All input beams of the amplifier are considered to have a Gaussian shape in space and time with different beam radii. The radius of the pump beam is assumed to have a value of 3 (arb. units) and the radius of the $2^{\text {nd }}$ Stokes beam is optimized to be half of the pump radius. 


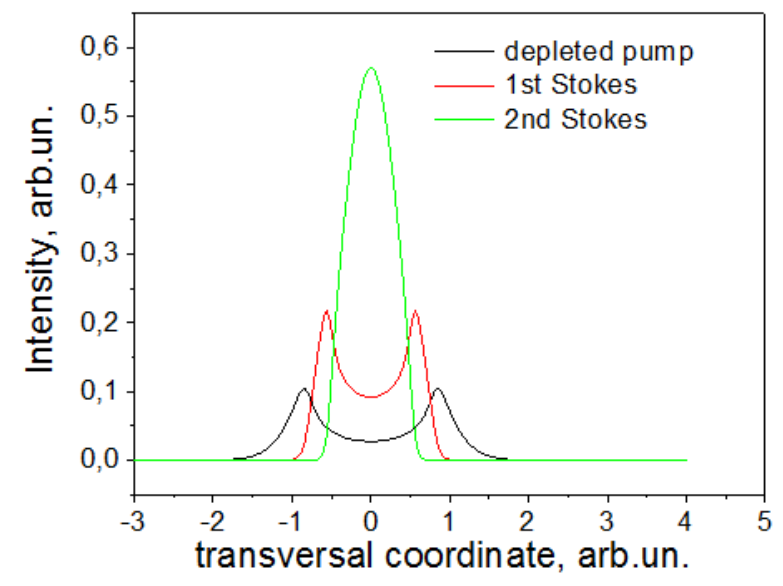

Figure 4. Cross-section of the depleted pump beam, and of the $1^{\text {st }}$ and $2^{\text {nd }}$ Stokes beams of a simultaneous Raman amplifier. ${ }^{12}$

The scheme of the simultaneous two-Stokes Raman amplifier with collinear propagation of waves in the same direction will be implemented. The principal setup of such a scheme is shown in figure 5. In particular, this means that if a nearly diffraction-limited ( $M^{2}$ factor close to 1) $1^{\text {st }}$ Stokes beam (the Stokes seed) is input into a Raman amplifier, the output Stokes beam is also nearly diffraction-limited even in the case of low beam quality pump radiation. The Raman amplification in crystals has been also demonstrated. ${ }^{11}$ As a result low divergent $\left(M^{2}\right.$ factor of 2.2$) 1^{\text {st }}$ Stokes radiation with pulse energy of $63 \mathrm{~mJ}$ was obtained.

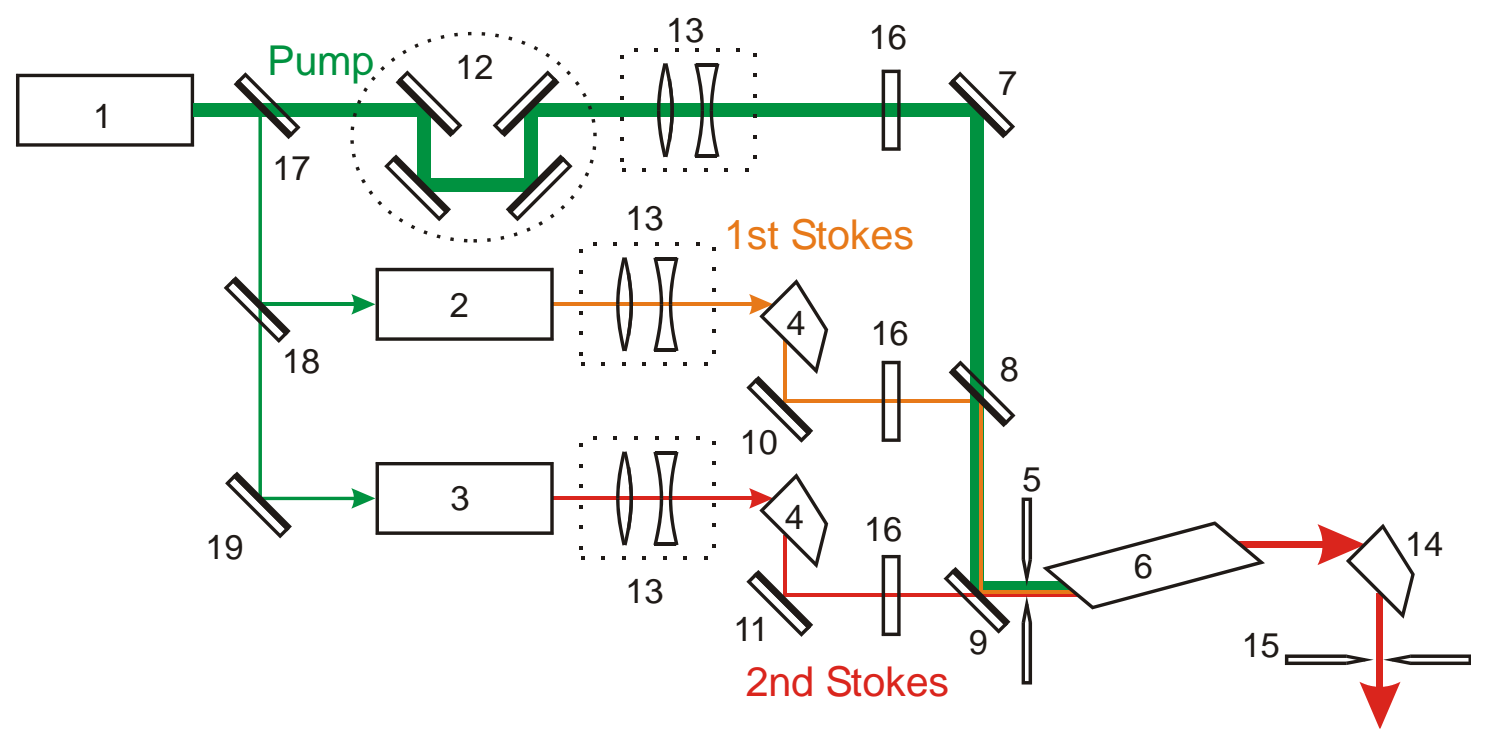

Figure 5. Planned setup of a simultaneous two-Stokes Raman amplifier: (1) is the Q-switched Nd:YAG laser with second harmonic generation; (2) is the $1^{\text {st }}$ Stokes Raman generator; (3) is the $2^{\text {nd }}$ Stokes Raman generator; (4) and (14) are Pellin-Broca prisms; (5) and (15) are apertures; (6) is the crystal of the Raman amplifier; (7)-(9) are mirrors for combining the pump wave with the $1^{\text {st }}$ and $2^{\text {nd }}$ Stokes waves; $(10)$ and $(11)$ are mirrors highly reflective at the $1^{\text {st }}$ and $2^{\text {nd }}$ Stokes wavelength, respectively; (12) is the time delay line; (13) are telescopes; (16) are attenuators; (17)-(19) are mirrors for splitting the pump radiation into three unequal parts. 


\section{CONCLUSION}

Over the last two decades, more than 130 crystals, ceramics, and glasses have been investigated including nitrates, tungstates, molybdates, borates, organics, laser host crystals, frequency doublers, silicon oxide, diamond, and silicon. Well above 2000 SRS emission wavelengths were observed when pumping the various Raman materials with Nd:YAG pump lasers and higher harmonics. $145 \mathrm{SRS}$ active vibration modes from $80 \mathrm{~cm}^{-1}$ to $3150 \mathrm{~cm}^{-1}$ were found. A current research project is presented, developing a dual frequency Raman amplifier system for the detection of ozone by differential absorption LIDAR (DIAL) systems.

\section{ACKNOWLEDGEMENT}

The author would like to thank Prof. Dr. A.A. Kaminskii for providing the $\mathrm{LuAlO}_{3}$-crystal used for the present study. We would like to thank the German Research Council, which funded this work under contract no. SCHR 462/11-1.

\section{REFERENCES}

[1] Woodbury, E. J. and Ng W. K., "Ruby Laser Operation in the Near IR", Proc. IRE 50, 2367 (1962).

[2] Eckhard, G., Hellwarth, R. W., McClung, F. J., Schwarz, S. E., Weiner, D. and Woodbury, E. J., "Stimulated Raman Scattering From Organic Liquids", Phys. Rev. Lett. 9, 455 (1962).

[3] Kaminskii, A. A., "Three-phonon stimulated Raman scattering in an orthorhombic LuAlO3 crystal", Dokl. Phys. 61, 586 (2016).

[4] Lux, O., Rhee, H., Fritsche, H., and Eichler, H. J., "Barium nitrate Raman laser at $1.599 \mu \mathrm{m}$ for $\mathrm{CO}_{2}$ detection", Proc. SPIE 8677, 86771B (2013).

[5] Rhee, H., "Stimulated Raman Scattering Spectroscopy in Crystalline Materials and Solid State Raman Lasers", dissertation TU Berlin, 2012.

[6] Lux, O., Fritsche, H., and Eichler, H. J., "Trace Gas Remote Sensing by Lasers", Optik \& Photonik 8, 48 (2013).

[7] Murray, J. T., Austin, W. L., and Powell, R. C., "Intracavity Raman conversion and Raman beam cleanup", Opt. Mater. 11, 353 (1999).

[8] Williams, R. J., Kitzler, O., Bai, Z., Sarang, S., Jasbeer, H., McKay, A., Antipov, S., Sabella, A., Lux, O., Spence, D. J., and Mildren, R. P., "High power diamond Raman lasers", IEEE J. Select. Topics Quantum Electron. 24, 1602214 (2018).

[9] Lux, O., Sarang, S., Williams, R. J., McKay, A., and Mildren, R. P., "Single longitudinal mode diamond Raman laser in the eye-safe spectral region for water vapor detection", Opt. Express 24, 27812 (2016).

[10]Rong, H. S., Xu, S. B., Cohen, O., Raday, O., Lee, M., Sih, V., and Paniccia, M., "A cascaded silicon Raman laser", Nat. Photonics 2, 170 (2008).

[11]Dong, B., Hu, J., Chen, Z., Yu, C., "Long-distance fiber sensor system based on the second-order Raman pump and amplification" Applied Physics B, 2012. 108(1): p. 57-60.

[12]Lisinetskii, V.A., V.A. Orlovich, H. Rhee, X. Wang, and H.J. Eichler, "Efficient Raman amplification of low divergent radiation in barium nitrate crystal”, Appl. Phys. B 91, 299 (2008). 